\title{
Accelerated Accumulation of Misfolded Prion Protein and Spongiform Degeneration in a Drosophila Model of Gerstmann-Sträussler-Scheinker Syndrome
}

\author{
Brendan A. Gavin, ${ }^{1}$ Maria J. Dolph, ${ }^{1}$ Nathan R. Deleault, ${ }^{2}$ James C. Geoghegan, ${ }^{2}$ Vikram Khurana, ${ }^{3}$ Mel B. Feany, ${ }^{3}$ \\ Patrick J. Dolph, ${ }^{1}$ and Surachai Supattapone ${ }^{2}$ \\ ${ }^{1}$ Department of Biology, Dartmouth College, Hanover, New Hampshire 03755, 22Department of Biochemistry, Dartmouth Medical School, Hanover, New \\ Hampshire 03755, and ' ${ }^{2}$ epartment of Pathology, Division of Neuropathology, Brigham and Women's Hospital and Harvard Medical School, Boston, \\ Massachusetts 02115
}

\begin{abstract}
Prion diseases are CNS disorders that can occur in sporadic, infectious, and inherited forms. Although all forms of prion disease are associated with the accumulation of pathogenic conformers of the prion protein, collectively termed $\operatorname{PrP}^{\mathrm{Sc}}$, the mechanisms by which $\mathrm{PrP}^{\mathrm{Sc}}$ molecules form and cause neuronal degeneration are unknown. Using the bipartite galactosidase-4 - upstream activating sequence expression system, we generated transgenic Drosophila melanogaster heterologously expressing either wild-type (WT) or mutant, disease-associated (P101L) mouse PrP molecules in cholinergic neurons. Transgenic flies expressing neuronal P101L PrP molecules exhibited severe locomotor dysfunction and premature death as larvae and adults. These striking clinical abnormalities were accompanied by age-dependent accumulation of misfolded PrP molecules, intracellular PrP aggregates, and neuronal vacuoles. In contrast, transgenic flies expressing comparable levels of WT PrP displayed no clinical, pathological, or biochemical abnormalities. These results indicate that transgenic Drosophila expressing neuronal P101L PrP specifically exhibit several hallmark features of human GerstmannSträussler-Scheinker (GSS) syndrome. Because the rates of abnormal PrP accumulation and clinical progression are highly accelerated in Drosophila compared with the rates of these processes in rodents or humans, the P101L mutant may be used for future genetic and pharmacologic studies as a novel invertebrate model of GSS.
\end{abstract}

Key words: prion; Drosophila; Gerstmann-Sträussler-Scheinker syndrome; PrP; neurodegeneration; misfolding

\section{Introduction}

Prion diseases are an unusual group of fatal neurodegenerative diseases that can occur in sporadic, infectious, or inherited forms (Glatzel et al., 2005). All forms of prion disease are associated with misfolding of the host-encoded prion protein, $\operatorname{PrP}^{\mathrm{C}}$, into one of several pathogenic isoforms, collectively termed $\operatorname{PrP}^{\mathrm{Sc}}$ (Prusiner, 1982). Approximately 10\% of human cases have a genetic origin, and all of these cases appear to be autosomal dominant disorders caused by various mutations in the coding sequence of the gene encoding $\operatorname{PrP}^{\mathrm{C}}$ (PRNP) (Palmer and Collinge, 1993; Prusiner, 1997). Remarkably, in several types of inherited prion disease, such as Gerstmann-Sträussler-Scheinker (GSS) syndrome, disease can be transmitted by inoculation of brain

\footnotetext{
Received April 6, 2006; revised Sept. 22, 2006; accepted 0ct. 20, 2006.

This work was supported in part by the National Institutes of Health and the Burroughs Welcome Fund. The confocal microscope used for this study was supported in part by National Science Foundation Grant DBI-9970048 (R.D.S.). We thank Mark McPeek for assistance with statistical analysis; Alex Raeber, Francisza Kuhn, and Bruno Oesch (Prionics, Schlieren, Switzerland) for kindly providing the 15B3 immunoprecipitation kit; and Judy Rees for help editing this manuscript.

Correspondence should be addressed to either of the following: Surachai Supattapone, Department of Biochemistry, Dartmouth Medical School, 7200 Vail Building, Hanover, NH 03755, E-mail: supattapone@Dartmouth.edu or Patrick J. Dolph, Department of Biology, Dartmouth College, Hanover, NH 03755, E-mail: partick.j.dolph@ dartmoth.edu.

DOI:10.1523/JNEUROSCI.3372-06.2006

Copyright $\odot 2006$ Society for Neuroscience $\quad$ 0270-6474/06/2612408-07\$15.00/0
}

homogenate from affected patients into normal primates and rodents harboring wild-type (WT) PRNP genes (Brown et al., 1994; Tateishi and Kitamoto, 1995). GSS can be caused by a variety of point or insertional mutations of the PRNP gene, but by far the most common mutation identified among affected families is $\mathrm{P} 102 \mathrm{~L}-129 \mathrm{M}$, in which the pathogenic PRNP haplotype contains the point mutation proline $\rightarrow$ leucine at codon 102 and methionine at the polymorphic codon 129 (Ghetti et al., 2003). In humans, P102L-129M GSS is characterized clinically by progressive ataxia and dementia, and pathologically by deposits of $\operatorname{PrP}$ amyloid plaques in the brain (Ghetti et al., 2003).

Although studies in transgenic ( $\mathrm{Tg}$ ) mice have shown that mutant $\operatorname{PrP}^{\mathrm{Sc}}$ conformers are primarily responsible for causing the pathological disease process in GSS (Hsiao et al., 1990; Manson et al., 1999; Nazor et al., 2005), it is unclear by what mechanisms those conformers are generated over time and how they produce neurological symptoms. To study these questions, it would be desirable to produce a GSS model in a system amenable to genetic analysis, ideally with a much shorter disease duration than observed in Tg mice and human patients. An attractive system that has been used successfully to model other human neurodegenerative diseases is the fruit fly, Drosophila melanogaster (Driscoll and Gerstbrein, 2003). Some advantages of Drosophila as a model organism include: short generation time, a well char- 
acterized genome containing many genes conserved in humans, and the availability of powerful, validated genetic techniques.

The Drosophila genome does not contain any endogenous PRNP or homologous genes. Previously, we and others have successfully produced Tg flies expressing WT PrP in neurons (Raeber et al., 1995; Deleault et al., 2003b). As anticipated, these $\mathrm{Tg}(\mathrm{WT}$ PrP) flies displayed no clinical or pathological abnormalities. In parallel, we also attempted to express PG14, an insertional PrP mutant containing 14 octapeptide repeats (Duchen et al., 1993; Krasemann et al., 1995; Chiesa et al., 2000), in Drosophila brain, but discovered that brain neurons possess a posttranscriptional mechanism that specifically suppresses the accumulation of PG14 PrP molecules (Deleault et al., 2003b). Consequently, $\operatorname{Tg}$ (PG14 PrP) flies displayed no behavioral or neuropathological abnormalities. In contrast, we show here that Drosophila neurons do not appear to suppress the accumulation of misfolded PrP P101L-128M (the murine homolog of human PrP P102L-129M) molecules. Furthermore, expression of PrP P101L molecules in Drosophila neurons produces a disease that recapitulates the major clinical, pathological, and biochemical features of GSS in human patients.

\section{Materials and Methods}

Fly stocks and plasmids. The galactosidase-4 (GAL4) driver lines w; $\mathrm{P}\{\mathrm{w}[+\mathrm{mC}]=$ Cha-GAL4.7.4 $\} 19 \mathrm{~B} \mathrm{P}\{\mathrm{w}[+\mathrm{mC}]=$ upstream activating sequence (UAS)-GFP. S65T\}T2, abbreviated as GAL4-Cha2, and $\mathrm{w}[1118] ; \mathrm{P}\{\mathrm{w}[+\mathrm{mC}]=$ Ddc-GAL4. $\mathrm{L}\} 4.36$, abbreviated as GAL4-Ddc, were obtained from the Bloomington Stock Center (Bloomington, IN). The third chromosome Cha insert line (GAL4-Cha3) was obtained from P. Salvaterra (Beckman Research Institute, Duarte, CA). The wild-type PrP plasmid psP72(MoPrP) was obtained from Mike Scott [University of California, San Francisco (UCSF), San Francisco, CA], and the transgenic line expressing wild-type $\operatorname{PrP}[\mathrm{P} 1]$ has been described previously (Deleault et al., 2003). The pSP72-MoPrP P101L plasmid was provided by S. B. Prusiner (UCSF, San Francisco, CA). The Drosophila transformation vector was obtained from Sharon Bickel (Dartmouth College, Hanover, NH). Unless otherwise indicated, all fly lines and stocks were maintained at room temperature (RT). Very rarely, flies carrying both the P101L and GAL4-Cha2 transgenes displayed no phenotype and did not express any PrP or green fluorescent protein (GFP). These animals were identified by fluorescence microscopy and excluded from additional study. Flies used for the lifespan study were kept at $25^{\circ} \mathrm{C}$ and placed in fresh food vials every other day.

Construction of DNA plasmids and transgenic fly lines. Activator constructs were created by separately ligating digested pUAST vector plasmid to wild-type (PRNP) mouse PrP or mutant (P101L) inserts, as described previously (Deleault et al., 2003a). The newly synthesized plasmids were purified by Qiagen (Hilden, Germany) Maxiprep columns and then used for the generation of the transgenic flies. All restriction enzymes were purchased from New England Biolabs (Beverly, MA).

The flies used for the generation of transgenic lines were $w$ to aid in the selection of transformants, and transgenic flies were generated by P-element induced transposition (Rubin and Spradling, 1982, 1983). The P101L transgenic flies used in this study contained either the P101L insert on the second $\left(\mathrm{P} 101 \mathrm{~L}^{\mathrm{A}}\right)$ or the third chromosome $\left(\mathrm{P} 101 \mathrm{~L}^{\mathrm{D}}\right)$. Homozygous or balanced activator lines were generated by standard genetic techniques and crossed to one of several tissue-specific driver lines to drive expression of PrP protein (Phelps and Brand, 1998). GAL4-Cha2 and GAL4-Cha3 were used to drive expression of $\operatorname{PrP}$ in cholinergic neurons, whereas GAL4-Ddc was used to drive expression of $\operatorname{PrP}$ in dopaminergic neurons.

Electrophoresis and immunoblotting. Homogenates were prepared by homogenizing 10 fly heads in $15 \mu$ l of lysis buffer (50 mu Tris, $\mathrm{pH} 7.5,10$ $\mathrm{mm} \mathrm{NaCl}, 0.5 \%$ Triton X-100, $0.5 \%$ deoxycholate) in a $0.2 \mathrm{ml}$ Kontes Micro glass tissue grinder. Fresh homogenates were used for all experiments. An equal volume ( $15 \mu \mathrm{l}$ ) of SDS loading buffer (containing $0.2 \mathrm{M}$ dithiothreitol) was added to each sample, which was then boiled at $95^{\circ} \mathrm{C}$ for $5 \mathrm{~min}$. SDS-PAGE was performed on $1.5 \mathrm{~mm}, 12 \%$ acrylamide gels with a ratio of 29:1 acrylamide/bis-acrylamide. After electrophoresis, proteins were blotted onto charged polyvinylidene difluoride membranes (Millipore, Bedford, MA) using a Fisher Biotech (Wembley, Australia) Semi Dry Blotting Unit transfer apparatus set at $0.3 \mathrm{~A}$ for $1 \mathrm{~h}$ and 15 min. After transfer, membranes were pretreated with 3 m guanidine isothiocyanate for $30 \mathrm{~min}$ and then rinsed for $1 \mathrm{~min}$ in distilled water followed by a $1 \mathrm{~min}$ rinse in PBST ( $1 \times$ PBS, $0.1 \%$ Tween 20$)$. The membrane was blocked using 5\% milk in PBST for $20 \mathrm{~min}$ at room temperature. Blocked membranes were incubated with anti-PrP D13 humanized Fab antibody fragment (kindly provided by Prof. S. B. Prusiner) diluted $1: 4000$ in $5 \%$ milk/PBST overnight at $4^{\circ} \mathrm{C}$. After incubation with primary antibody, membranes were washed three times for 10 min in PBST, incubated with horseradish peroxidase (HRP)-labeled anti-human Fab2 conjugate (Pierce, Rockford, IL), diluted 1:20,000 in 5\% milk/PBST for $1 \mathrm{~h}$ at RT, and washed again three times for $10 \mathrm{~min}$ in PBST. After chemiluminescent development with Pico SuperSignal ECL reagent (Pierce) for $1 \mathrm{~min}$, blots were exposed to BioMax film (Kodak, Rochester, $\mathrm{NY}$ ), and films were processed in a Kodak M35A X-OMAT film processor. The ECL Advance Detection kit (GE Healthcare, Arlington Heights, IL) was used for the detection of PrP in GAL4-Ddc flies, because of low expression levels of PrP protein in these lines. Prestained protein standards (Invitrogen, Eugene, OR) were used to indicate the apparent molecular weights of proteins.

Proteinase $K$ digestion assay. Proteinase $\mathrm{K}(\mathrm{PK})$ digestion was performed as described previously (Tremblay et al., 2004). Thirty-five heads from $\sim 3$-week-old flies were homogenized in $40 \mu \mathrm{lof} \mathrm{Ca}^{2+} / \mathrm{Mg}^{2+}$-free PBS. Homogenates were briefly sonicated and spun at $2000 \mathrm{rpm}$ for 1 min to remove cuticle. It was determined previously that $10 \mu \mathrm{g} / \mathrm{ml} \mathrm{PK}$ was the minimum concentration necessary for complete digestion of WT PrP. Thus, to examine the sensitivity of P101L to PK digestion, all homogenates were incubated with $10 \mu \mathrm{g} / \mathrm{ml}$ PK (Roche, Welwyn Garden City, UK) and $1 \%$ nonidet $\mathrm{P}-40$ for $1 \mathrm{~h}$ at $4^{\circ} \mathrm{C}$ with periodic mixing. The digestion was quenched by adding $200 \mu \mathrm{g} / \mathrm{ml}$ of aprotinin and leupeptin (Sigma, St. Louis, MO) and $5 \mathrm{~mm}$ phenylmethylsulfonylfluoride. The inactivated samples received an equal volume of SDS loading buffer and were analyzed by Western blot analysis.

Detergent solubility assay. To test for solubility differences between Drosophila-expressed WT and P101L PrP, 40 heads from 3-week-old flies were homogenized in $40 \mu \mathrm{l}$ of Sarkosyl buffer (2\% Sarkosyl, $50 \mathrm{~mm}$ Tris, $\mathrm{pH} 7.5,10 \mathrm{~mm} \mathrm{NaCl})$. Homogenates were sonicated briefly and spun at $2000 \mathrm{rpm}$ for $1 \mathrm{~min}$ to remove cuticle. Supernatants were removed and incubated for $1 \mathrm{~h}$ at $4^{\circ} \mathrm{C}$ with periodic mixing. After incubation, $20 \mu \mathrm{l}$ from each sample were removed and spun at $100,000 \times g$ for $1 \mathrm{~h}$ at $4^{\circ} \mathrm{C}$. The soluble supernatant fraction was removed from each sample and the insoluble pellet fraction was resuspended in $20 \mu \mathrm{l}$ of the original Sarkosyl buffer. All samples were then analyzed by Western blot.

$15 B 3$ immunoprecipitation assay. Immunoprecipitation of the misfolded form of P101L was performed using a modification of the protocol described by Nazor et al. (2005). The 15B3 antibody recognizes specifically $\operatorname{PrP}^{\text {Sc }}$ (Korth et al., 1997) and was generously provided by Alex Raeber, Franziska Kuhn, and Bruno Oesch (Prionics AG, Schlieren, Switzerland) along with all required buffer solutions. Three hundred microliters of Dynabeads M-450 rat anti-mouse IgM (Invitrogen) were resuspended in $1.5 \mathrm{ml}$ of Prionics coating buffer and incubated with $10 \mu \mathrm{g}$ of $15 \mathrm{~B} 3$ antibody for $2 \mathrm{~h}$ at RT on a rotating wheel. Beads were then washed three times in coating buffer. Either 80 or 40 fly heads from each genotype were homogenized in $50 \mu \mathrm{l}$ of Prionics homogenization buffer, as indicated. Twenty microliters of sample then were incubated with $50 \mu \mathrm{l}$ of coated beads and $1 \mathrm{ml}$ of Prionics immunoprecipitation buffer for $1 \mathrm{~h}$ on a rotating wheel at RT. The beads were washed three times using the Prionics washing buffer. Immunoprecipitated proteins were clarified by electrophoresis and immunoblotting as described above. Membranes were blocked using $2 \%(\mathrm{w} / \mathrm{v})$ blocking solution provided in the ECL Advance Detection kit (GE Healthcare) in PBST. Immunoprecipitated PrP was detected on membranes using the Prionics monoclonal antibody (Mab) 6H4 conjugated to HRP at a 1:5000 dilution in PBST, developed using the ECL Advance Detection kit (GE Healthcare), and exposed to BioMax chemiluminescent film (Kodak, Cedex, France). 
Larval brain dissection and immunofluorescence studies. Brains and spinal cords from third instar larvae were dissected in standard saline buffer solution [containing the following (in $\mathrm{mM}$ ): $2 \mathrm{KCl}, 128 \mathrm{NaCl}, 80 \mathrm{MgCl}_{2}$, $1.8 \mathrm{CaCl}_{2}, 36$ sucrose, and 5 HEPES, $\left.\mathrm{pH} 7.1\right]$ and then fixed in $4 \%$ paraformaldehyde in microtubule-stabilizing buffer ( 5 mM EGTA, 2 mM $\left.\mathrm{MgCl}_{2}\right)$ for $15 \mathrm{~min}$ at $4^{\circ} \mathrm{C}$. Samples were washed six times in PBS-TX $(1 \times$ PBS plus $0.3 \%$ Triton-X 100) and incubated in AH6 anti-PrP antibody (TSE Reagent Resource Center, Compton, UK) diluted 1:50 in PBS-TX plus $5 \%$ fetal calf serum (FCS) overnight at $4^{\circ} \mathrm{C}$. After another six washes in PBS-TX, samples were incubated in Texas Red goat anti-mouse (Jackson Immunologicals, West Grove, PA) in PBS-TX plus 5\% FCS overnight. After three washes in PBS, samples were mounted in Prolong Antifade media (Invitrogen) and examined using a Leica (Nussloch, Germany) confocal microscope (model TCS SP UV). Image analysis was performed using Leica confocal software and Adobe (San Jose, CA) Photoshop.

Neuropathology. Larval brains were sectioned and immersed in $0.1 \mathrm{M}$ sodium phosphate buffer, $\mathrm{pH} 7.2$, containing $2 \%$ glutaraldehyde (Ladd Research, Williston, VT) and $2 \%$ osmium tetroxide $\left(\mathrm{OsO}_{4}\right)$ (Ladd Research) for $30 \mathrm{~min}$ on ice. Glutaraldehyde/OsO $\mathrm{O}_{4}$ solution was removed and eyes were rinsed in $0.1 \mathrm{M}$ sodium phosphate buffer, $\mathrm{pH}$ 7.2. Fresh $2 \%$ $\mathrm{OsO}_{4}$ was added and brains were incubated for $2 \mathrm{~h}$ at $4^{\circ} \mathrm{C}$. Osmium was removed and a standard ethanol dehydration series was performed in 30, 50, 70, 90\% (once), and absolute (twice) EtOH using 10 min washes. Brains were equilibrated twice in propylene oxide (Ladd Research) and infused in 50\% Durcupan resin (Sigma)/50\% propylene oxide overnight. The 50/50 Durcupan/propylene oxide mixture was removed and replaced with $100 \%$ Durcupan, and brains were incubated for an additional $4 \mathrm{~h}$ and then baked overnight in molds containing 100\% Durcupan. Using a Sorvall (Asheville, NC) MT5000 ultramicrotome, $0.5 \mu \mathrm{m}$ sections were cut and stained with toluidine blue solution ( $1 \%$ toluidine blue, $1 \%$ sodium borate in water) and viewed under a light microscope.

Heads from adult flies at 1,14 , and $28 \mathrm{~d}$ posteclosion were fixed in formalin, embedded in paraffin, and $4 \mu \mathrm{m}$ frontal sections were prepared. Serial sections were cut through the entire brain, and placed on a single glass slide. Hematoxylin and eosin staining was performed using a standard protocol. Vacuoles larger than $5 \mu \mathrm{m}$ were counted throughout the whole brain. At least eight individual hemibrains were analyzed per genotype and time point. Immunostaining on paraffin sections was performed using the avidin-biotin-peroxidase method after antigen retrieval with microwaving in $10 \mathrm{~mm}$ sodium citrate, $\mathrm{pH}$ 6.6. The AH6 monoclonal antibody was used at a dilution of 1:500. Counterstaining with hematoxylin was performed to highlight cortical nuclei.

Survival and climbing assays. Approximately 100 newly eclosed flies were collected from each genotype, placed into vials, and monitored daily for viability. For P101L ${ }^{\mathrm{A}}$;GAL4-Ddc flies, only 30 flies were collected for this survival assay. To prevent the lethargic P101L flies from getting trapped in their own food, the vials were changed to fresh food every other day. Locomotor function was assessed in flies using a climbing assay similar to that described previously (Ganetzky and Flanagan, 1978; Le Bourg and Lints, 1992). For each genotype tested, 30 individual flies were anesthetized using carbon dioxide and placed into empty plastic vials with a line drawn $4 \mathrm{~cm}$ from the bottom of each tube. After $\sim 10 \mathrm{~min}$ of recovery time from the anesthesia, flies were knocked to the bottom of the tube, and the ability of each fly to successfully climb above the $4 \mathrm{~cm}$ mark by $30 \mathrm{~s}$ was scored. Climbing assays were repeated 5-10 times for each fly.

\section{Results}

\section{Expression of murine prion protein in transgenic Drosophila} We generated transgenic Drosophila that contain either the wildtype (PRNP) or P101L mouse PrP genes, under the control of the UAS promoter. To drive expression of WT or P101L PrP specifically in cholinergic or dopaminergic neurons, each of the PrP activator lines was crossed with a driver line that carried either the GAL4-Cha or GAL4-Ddc transgene, respectively. PrP levels were measured in transgenic flies by Western blot, and we observed strong expression of prion protein in all crosses (Fig. $1 A, B$ ). The
A.

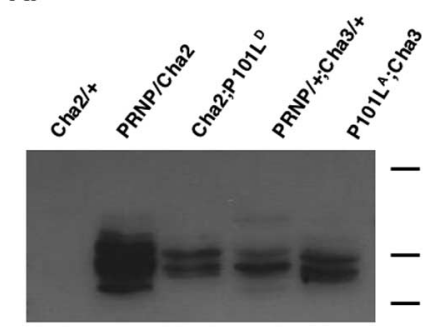

B.

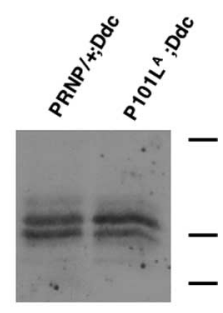

C.

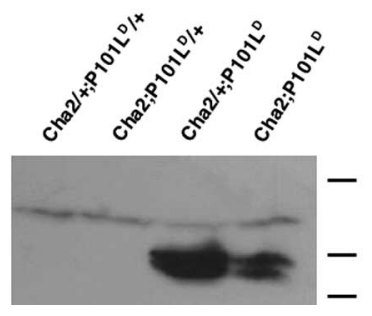

Figure 1. WT and P101L prion protein expression in the brains of transgenic Drosophila. Western blot of head homogenates from transgenic flies expressing either WT PrP or P101L in cholinergic neurons is shown, using D13 Fab to probe PrP levels. A, GAL4-Cha2, PRNP, and $\mathrm{P} 101 \mathrm{~L}^{\mathrm{A}}$ are second chromosomal inserts; GAL4-Cha3 and P101L ${ }^{\mathrm{D}}$ inserts are on the third chromosome. All flies were $\sim 1$ week old at the time of sample preparation. $\boldsymbol{B}$, The GAL4-Ddc insert is on the third chromosome. C, Two copies of P101L, but only one copy of GAL4-Cha, are required for P101L expression. Flies were $\sim 3 \mathrm{~d}$ old at the time of sample preparation. PRNP, P element containing a wild-type copy of mouse PRNP gene fused to the yeast UAS enhancer; P101L, P element containing a mutant copy of mouse PRNP gene, with proline to leucine substitution at codon 101, fused to the yeast UAS enhancer. Heterozygotes are indicated as transgene/+. Molecular weight bands indicate 40,25 , and $20 \mathrm{kDa}$.

Table 1. Neuronal expression of PrP results in larval lethality

\begin{tabular}{lc}
\hline & \% Eclosion \\
\hline Expected & 100 \\
P101L'; ${ }^{\mathrm{A}}$ (ha3 & 52.2 \\
Cha2;P101L $^{\mathrm{D}}$ & 53.1 \\
P101L;Ddc & 68.4
\end{tabular}

The percentage of eclosed P101L-expressing flies is shown. The percentage of flies is determined by dividing the observed number of eclosed P101L flies in a balanced stock by the predicted number of eclosed mutant flies in a balanced stock. At least 250 eclosed adults were scored in each fly line. Expected eclosion percentage represents predicted eclosion of GAL4;P101L transgenic flies from a balanced stock.

PrP banding pattern detected by immunoblot (Fig. 1) is consistent among all PrP-expressing fly lines. This banding pattern is not the result of differences in PrP glycosylation, as revealed by $\mathrm{N}$-glycosidase $\mathrm{F}$ digestion assays (data not shown). When expressing P101L in cholinergic neurons, we found that two copies of the P101L insert are necessary for high levels of expression. However, sufficient expression can be attained from a single copy of the GAL4-Cha driver (Fig. 1C). In cases in which P101L was expressed in dopaminergic neurons, only one copy of $\mathrm{P} 101 \mathrm{~L}^{\mathrm{A}}$ is necessary, but two copies of GAL4-Ddc are required for sufficient expression of protein (data not shown). To eliminate the possibility that any phenotypes are attributable to a general effect of protein over-expression, lines that expressed lower levels of P101L than WT PrP were chosen for the majority of subsequent experiments (PRNP/GAL4-Cha2 and GAL4-Cha2; P101L ${ }^{\mathrm{D}}$ ).

\section{P101L larvae display behavioral defects and aberrant neuronal PrP localization}

Expression of P101L, but not WT, PrP molecules in cholinergic neurons causes an abnormal phenotype that appeared in animals as young as third instar larvae. Specifically, we observed that some of these larvae crawl backwards along the sides of their vials 
A.

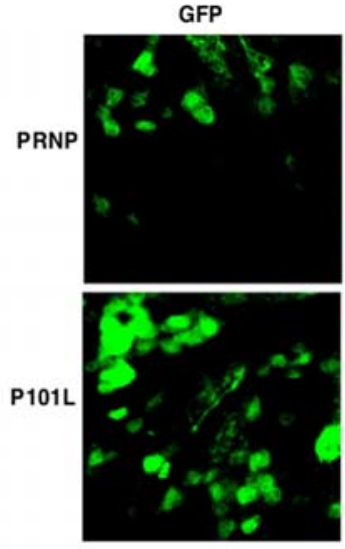

WT

B.

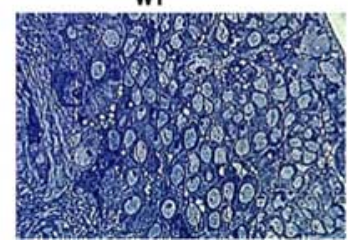

PrP

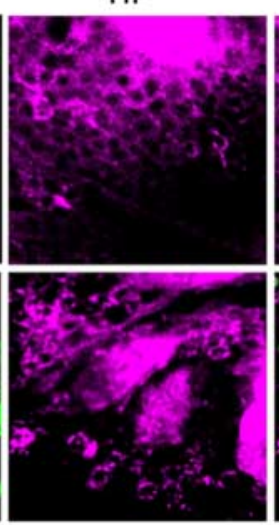

P101L

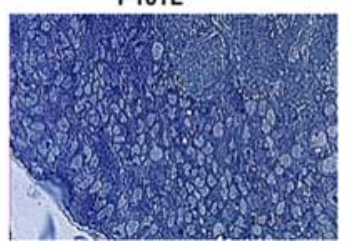

Merge
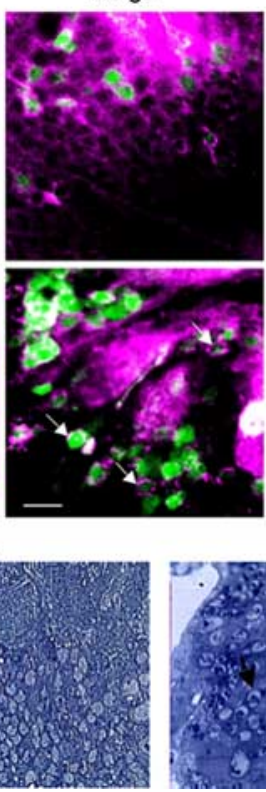

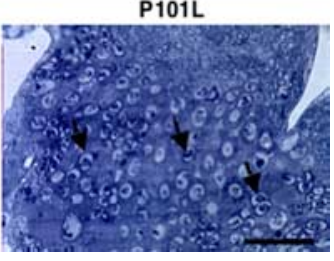

Figure 2. P101L Accumulates as aggregates in the brains of transgenic larvae. Immunological staining and ultrastructural analysis of larval brain sections is shown. $A$, Whole-mount third-instar larval brain samples were prepared, fixed, and stained with AH6 Mab, as described in methods. P101L larval brains were homozygous for the P101L insert, but may have carried either one or two copies of the GAL4-Cha2 driver. All PRNP flies were heterozygous for the PRNP insert and for the GAL4-Cha2 driver. Green fluorescence is caused by the expression of GFP in cholinergic neurons, whereas Texas Red fluorescence indicates PrP staining. PrP (Texas Red) is displayed as magenta to be red/green color blind compatible. The colocalization of GFP (green) and Texas Red appears as white. Higher magnification of GAL4-Cha2;P101L samples is shown in three far right panels. Arrows indicate aggregated PrP both in the cell periphery and cytoplasm. $\boldsymbol{B}$, WT or P101L PrP-expressing third-instar larval brain samples stained with toluidine blue. P101L (left) shows a healthy brain section, whereas in P101L (right) dark punctate staining is observed (arrows). Scale bars: $A, 10 \mu \mathrm{m} ; \boldsymbol{B}, 20 \mu \mathrm{m}$.

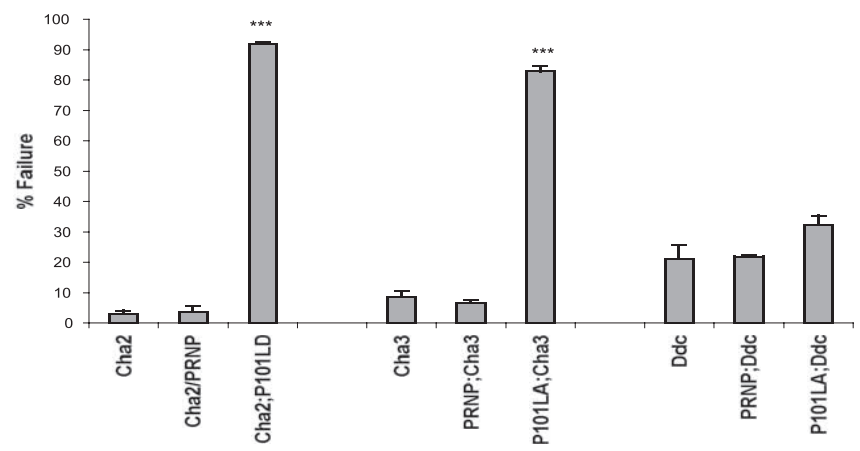

Figure 3. Climbing activity of adult P101L and WT PrP expressing transgenic flies. The climbing assay was measured as described in Materials and Methods using flies that were $<1 \mathrm{~d}$ old. Thirty flies from each group were examined in 10 consecutive climbing assay tests. Each bar represents the percentage of flies from each group that failed to climb $>4 \mathrm{~cm}$ in the climbing chamber within $30 \mathrm{~s}$. Asterisks indicate a significant difference between nontransgenic controls and PrP-expressing flies. PRNP;Cha3 and PRNP;Ddc were both heterozygous for PRNP. The $\chi^{2}$ value $(\mathrm{df}=1)=41.71$ with $p<0.0001$ using a contingency table analysis. NT, Nontransgenic controls; Cha2, GAL4-Cha2; Cha3, GAL4-Cha3; Ddc, GAL4-Ddc. Error bars indicate SEM.

and often stop to swing the anterior parts of their bodies. In addition, P101L larvae burrow through the culture media to the bottom of a vial, where they often died. Approximately half of the flies expressing P101L in cholinergic neurons did not eclose (Table 1), regardless of the GAL4-Cha driver line or P101L insert line used. To a lesser extent, larval lethality was also observed in animals expressing P101L in dopaminergic neurons (Table 1).

To further investigate these larval phenotypes, we examined the spatial expression of PrP in transgenic animals. The GAL4-Cha2 driver line also possesses a transgene on the second chromosome

groom their wings and antennae. To quantitate the locomotor defects associated with these P101Lexpressing flies, we used a variation of a climbing assay commonly used to assess mobility response and coordination in fruit flies (Ganetzky and Flanagan, 1978; Le Bourg and Lints, 1992). Drosophila is normally negatively geotactic and spends much of its captive life flying or climbing to the top of a vial (Ganetzky and Flanagan, 1978). However, between 82 and 94\% of flies expressing P101L in cholinergic neurons did not climb and often remained motionless for the duration of the experiment (Fig. 3). In contrast, flies expressing WT PrP in cholinergic neurons and nontransgenic animals climbed normally (Fig. 3 ). The finding that the expression of P101L PrP in cholinergic neurons results in severe locomotor defects regardless of the P101L chromosomal insert used $\left(\mathrm{P}_{101 \mathrm{~L}^{\mathrm{A}} \text { or P101L }}{ }^{\mathrm{D}}\right)$ effectively rules out the possibility that these observed phenotypes are attributable solely to insertion effects of the UAS-P101L transposon. Newly eclosed flies expressing P101L in dopaminergic neurons displayed mild coordination defects in that their legs often move in an uncontrolled, flailing motion. However, these P101L flies did not perform statistically worse then the PRNP;GAL4-Ddc flies or nontransgenic controls (Fig. 3).

To assess the effects of mutant prion expression on the survival of adult transgenic animals, we monitored the viability of P101L and WT PrP-expressing flies. The majority of P101L flies, again regardless of the P101L insert line used, died by 3-4 weeks compared with 8-10 weeks for flies expressing WT PrP or only GAL4-Cha or flies carrying, but not expressing, the P101L $\mathrm{L}^{\mathrm{D}}$ insert (Fig. 4). Therefore the expression of P101L in cholinergic neurons resulted in $\sim 50 \%$ reduction in lifespan. In comparison, $20-90 \%$ of flies expressing P101L in dopaminergic neurons were 
dead within $24 \mathrm{~h}$ posteclosion. We are currently investigating the basis for the rapid and variable lethality observed in animals with dopaminergic-specific expression of P101L PrP.

\section{Adult Drosophila expressing P101L exhibit an age-dependent neuropathology}

To study the neuropathology of adult transgenic P101L fly brains, we examined standard hematoxylin- and eosin-stained sections of 1-d-old and aged transgenic flies and nontransgenic controls. Flies expressing WT PrP showed only infrequent neuropil vacuoles (4.1 vacuoles/hemibrain) in aged animals (Fig. $5 A$, arrow). At $1 \mathrm{~d}$ of age, flies expressing P101L were also histologically normal (Fig. 5B). However, by day 28, flies expressing mutant PrP showed numerous neuropil vacuoles in the brains (16.5 vacuoles/ hemibrain), particularly in the optic lobes (Fig. $5 C$, arrows, $I$ ). To determine whether this age-dependent vacuolation in $\mathrm{P} 101 \mathrm{~L}-$ expressing flies was accompanied by apoptosis, we performed terminal deoxynucleotidyl transferase-mediated biotinylated UTP nick end labeling (TUNEL) analysis on sections of P101L and WT PrP-expressing flies. There was no significant difference in TUNEL staining between these two groups (data not shown). The lack of increased apoptosis in P101L-expressing flies parallels the situation in human GSS in which few apoptotic cells can be detected (Gray et al., 1999).

To localize wild-type and mutant prion protein in the adult fly brain, and to monitor changes in localization and accumulation over time, we immunostained sections from WT PrP and P101L-expressing transgenic animals at 1,14 , and $28 \mathrm{~d}$ of age. $\mathrm{PrP}$ accumulates over time in both lines of transgenic flies (Fig. 5A-G). In addition, P101L flies show a much more punctate pattern of immunoreactivity (Fig. $5 F, G$ ), particularly at older ages (Fig. 5G). At higher magnification, inclusions can be seen in neuronal cell bodies (Fig. $5 H$, arrow) and are often multiple. Punctate structures consistent with neuritic inclusions are also seen within the neuropil of P101L brains (Fig. 5H, arrowhead).

\section{Age-dependent accumulation of misfolded P101L PrP in transgenic Drosophila}

The 15B3 antibody is a monoclonal IgM antibody that specifically recognizes prion protein in its $\mathrm{PrP}^{\mathrm{Sc}}$ conformation (Korth et al., 1997) and that has been reported recently to immunoprecipitate a conformationally distinct isoform of PrP from P101L mouse brain homogenates (Nazor et al., 2005). We were able to immunoprecipitate 15B3-reactive PrP from 3-weekold P101L-expressing flies (Fig. 6A). Only trace amounts of this immunoprecipitable PrP was detected in age-matched WT PrPexpressing flies. These findings strongly suggest that the P101L expressed in transgenic Drosophila converts to a conformation distinct from WT PrP. In addition, we observed an age-dependent increase in 15B3-reactive PrP in P101L flies, with 2- to 3-week-old animals expressing signifiError bars indicate SD. cantly more than 1- to 2-d-old flies (Fig. 6B). Larvae expressing P101L were also found to contain low levels of 15B3-reactive PrP (data not shown).

We also tested the solubility of our WT and P101L prion protein in $2 \%$ Sarkosyl. We found no observable difference between the levels of detergent-insoluble PrP in WT and P101Lexpressing 3-week-old flies (Fig. 6C). The majority of $\mathrm{PrP}$ was found in the soluble fraction for both groups (Fig. 6C). In addition, we tested the protease sensitivity of our P101L prion protein using "cold" digestion (Tremblay et al., 2004) and found that P101L and WT PrP were equally protease sensitive (Fig. 6D),

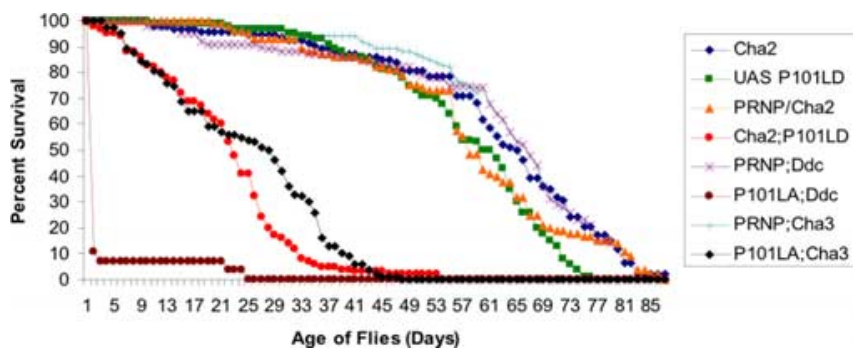

Figure 4. Survival curve of P101L and WT PrP-expressing transgenic flies. Flies from each genotype were maintained at $25^{\circ} \mathrm{C}$ in fresh food vials changed every other day for the duration of this study. The graph reflects the percentage of surviving animals with dead flies recorded daily. The PRNP/+;GAL4-Ddc and PRNP;Cha3 survival assays are ongoing experiments.
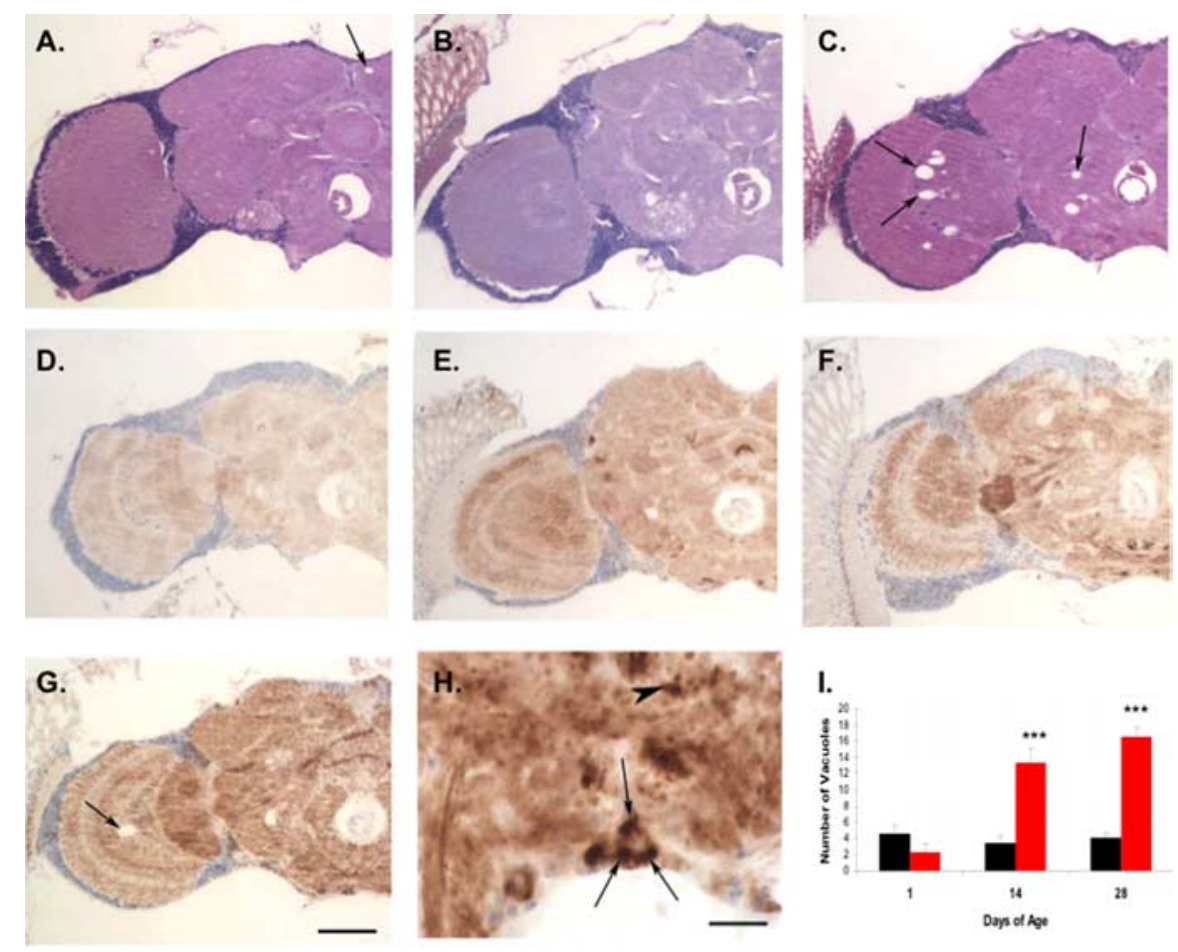

Figure 5. Neuropathology of adult P101L and WT PrP-expressing transgenic flies. $\boldsymbol{A}-\boldsymbol{C}$, Hematoxylin- and eosin-stained frontal sections from 1-d-old GAL4-Cha2 flies expressing WT PrP $(\boldsymbol{A})$ show minimal vacuolation (arrow). One-day-old GAL4-Cha2 flies expressing P101L (B) are also histologically normal. Twenty-eight-day-old flies expressing P101L show prominent vacuolar pathology (C, arrows). D-G, Flies expressing WT PrP show accumulation of prion protein from day $1(\boldsymbol{D})$ to day $28(\boldsymbol{E})$. Flies expressing P101L also accumulate prion protein from day $1(\boldsymbol{F})$ to day $28(\boldsymbol{G})$ and in addition show a more punctate pattern of immunoreactivity in both the neuropil and cell cortex. Note also the optic lobe vacuole in the aged P101L animal $(\boldsymbol{G}$, arrow). $\boldsymbol{H}, A t$ higher magnification, P101L flies show prominent intracellular inclusion formation in neuronal cell bodies (arrows) and punctate neuropil structures (arrowhead). Scale bars: (in $\mathbf{G}) \boldsymbol{A}-\mathbf{G}, 50 \mu \mathrm{m} ; \boldsymbol{H}, 10 \mu \mathrm{m}$. I, Average number of vacuoles observed in hemibrain sections from adult flies. Black bars, PRNP/GAL4-Cha2; red bars, GAL4-Cha2;P101L. Asterisks indicate significance for number of vacuoles; ${ }^{* * *} p<0.005$ and was determined using Excel software (Microsoft, Redmond, WA) with supplementary Student's t test. 
A.

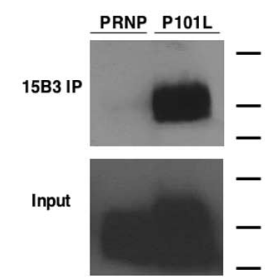

c.

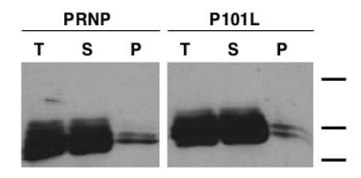

B.

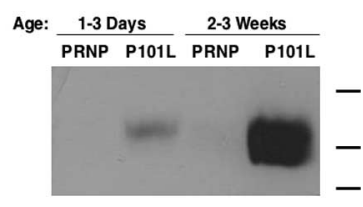

D.

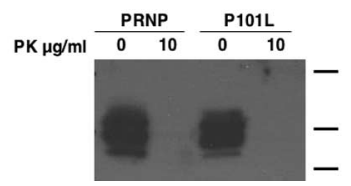

Figure 6. Transgenic flies accumulate misfolded P101L PrP in an age-dependent manner. Biochemical analysis of brain homogenate samples from transgenic GAL4-Cha2 flies is shown. All flies used in the experiments designed for the biochemical analysis of P101L PrP, unless stated otherwise, were at least 3 weeks in age. Molecular weight bands shown indicate 40, 25, and $20 \mathrm{kDa}$. $\boldsymbol{A}$, For each sample indicated, 80 fly heads were homogenized to generate each sample, and brain homogenates were subjected to $15 \mathrm{~B} 3$ immunoprecipitation according to the protocol described in Materials and Methods. The input reflects one-fourth of the total amount of protein loaded in the immunoprecipitation experiment. The Western blots were probed with a 6 H4-HRP conjugate. $\boldsymbol{B}$, For each sample indicated, 40 fly heads were homogenized and subjected to immunoprecipitation using the $15 B 3$ antibody. The Western blot was probed with a 6H4-HRP conjugate. C, Head homogenates were solubilized in 2\% Sarkosyl for $1 \mathrm{~h}$ followed by

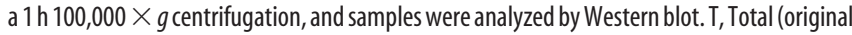
sample); S, soluble; P, pellet. D, Head homogenates were treated with or without $10 \mu \mathrm{g} / \mathrm{ml}$ PK for $1 \mathrm{~h}$ at $4^{\circ} \mathrm{C}$ and subsequently analyzed by Western blot using Fab D13.

suggesting that the P101L mutation did not confer protease resistance.

\section{Discussion}

To study the relationship between mutant PrP molecules and disease pathogenesis in GSS, several groups have previously created transgenic mice expressing MoPrP P101L, the mouse homolog of human PrP P102L (Hsiao et al., 1990; Manson et al., 1999; Nazor et al., 2005). These studies showed that, although Tg mice expressing MoPrP P101L molecules at levels similar to endogenous WT PrP did not any display clinical, pathological, or biochemical abnormalities, $\operatorname{Tg}(\mathrm{MoPrP}$ P101L) mice expressing higher levels of the mutant protein developed a disease resembling GSS. Strikingly, the age-dependent development of clinical symptoms in $\mathrm{Tg}(\mathrm{MoPrP} \mathrm{P} 101 \mathrm{~L})$ high expressor mice was accompanied by the accumulation of amyloid deposits in the brain and the conversion of mutant PrP into a conformer that could be immunoprecipitated by a $\mathrm{PrP}^{\mathrm{Sc}}$-specific Mab, $15 \mathrm{~B} 3$ (Nazor et al., 2005). Moreover, the GSS-like disease observed in $\mathrm{Tg}(\mathrm{MoPrP}$ P101L) high expressor mice can be accelerated by inoculation of brain homogenates from other, symptomatic $\operatorname{Tg}(\mathrm{MoPrP}$ P101L) mice (Nazor et al., 2005).

By expressing P101L PrP in transgenic Drosophila, we have generated an invertebrate model of prion disease, which manifests clinical, pathological, and biochemical correlates to human GSS. This Drosophila model mimics the symptoms associated with human cases of GSS in several ways. First, both human GSS patients and P101L flies display significant locomotor defects and shortened lifespans. Second, age-dependent vacuolation and accumulation of PrP aggregates are observed in both symptomatic Drosophila P101L and human GSS brain sections (Sasaki et al., 2003). However, it should be noted that although spongiform appearance is a neuropathological hallmark of prion diseases in mammals, neurodegeneration in a number of Drosophila neurodegenerative disease models is associated with vacuolation (Witt-

mann et al., 2001). The mechanisms involved in producing spongiform degeneration in prion diseases are currently unknown. Botella et al. (2003) reported that Ras signaling plays an important role in the formation of vacuoles in Drosophila brain. It is interesting to speculate that the Ras signaling system might specifically play a role in the formation of vacuoles in P101L flies, but more work is required to test this hypothesis. A final similarity between human cases of GSS disease and transgenic P101L flies is that 15B3-reactive mutant PrP molecules can be detected in symptomatic brain tissue.

In considering the characteristics of GSS in human, mouse, and Drosophila, it is interesting to note that the rate of clinical progression correlates with the lifespan of the animal host. Humans do not typically exhibit symptoms of GSS disease or show signs of neuropathology until $\sim 50$ years of age, transgenic mice expressing P101L do not develop symptoms until $\sim 6$ months of age (Hsiao et al., 1990; Nazor et al., 2005), and the majority of transgenic P101L-expressing Drosophila die 3-4 weeks after eclosion. This acceleration of disease onset is not surprising, because similar findings have also been observed in several other Drosophila models of human neurodegenerative diseases (Warrick et al., 1998; Feany and Bender, 2000; Marsh et al., 2000; Wittmann et al., 2001). The mechanism by which misfolded PrP molecules accumulate $\sim 10$-fold more rapidly in flies than in mice is currently unclear, but may be the result of an age-dependent increase in the formation of 15B3-reactive PrP or to the inability of older neurons to clear misfolded PrP.

Several differences between the P101L transgenic fly model and human cases of GSS disease were detected in this study. First, although misfolded PrP is typically characterized as being detergent insoluble and protease resistant, we were unable to isolate detergent-insoluble or PK-resistant P101L PrP from our fly brain homogenates. The absence of insoluble P101L is not surprising, as this was shown previously in the transgenic mouse model for GSS disease (Nazor et al., 2005), and the absence of protease resistant P101L PrP may be attributable to processing differences of this protein in transgenic fly brains compared with brains of mice. A second surprising feature observed in P101L-expressing animals was the appearance of locomotor defects and lethality in animals as young as second or third instar P101L larvae. It is possible that low levels of misfolded $\mathrm{PrP}$ molecules are toxic to some larvae. It is also currently unclear why genetically identical animals (GAL4-Cha2; P101L ${ }^{\mathrm{D}}$ ) do not die at a uniform time point (Fig. 4), but these results are likely attributable to the complex processes of protein clearance and degradation that may vary from fly to fly. A final unexpected finding was that flies expressing P101L PrP in cholinergic neurons display a different phenotype from animals expressing P101L PrP in dopaminergic neurons. Cholinergic neurons serve important roles in motor control and, thus, it is not surprising to find that coordination and motor skills were compromised in flies expressing P101L in this subset of neurons. However, it is unclear why expression of $\mathrm{P} 101 \mathrm{~L}$ in dopaminergic neurons results in a distinct new behavioral phenotype and in drastically shortened lifespans. It is possible that P101L expression results in neuronal loss of function, and that dopaminergic neurons are more essential than cholinergic neurons to the survival of the organism.

An invertebrate model of prion disease offers several advantages as a research tool. The short lifespan and generation time of Drosophila, combined with the rapid onset of disease observed in P101L transgenic flies, should accelerate the pace of experimentation for future studies. In addition, the availability of numerous mutant fruit fly stocks and the malleability of the Drosophila ge- 
nome will facilitate the design of genetic screens for modifiers of the P101L phenotype.

Transgenic P101L flies could also be used to screen for prophylactic compounds, which might prevent the progression of GSS and other inherited prion diseases in human patients. Unlike patients with sporadic or infectious forms of prion disease, patients harboring pathogenic PrP mutations can be identified at birth by DNA testing. Therefore, a unique opportunity exists in this particular group of patients to administer treatments early in the disease process, before the onset of protein misfolding, neurodegeneration, and clinical deterioration.

\section{References}

Botella JA, Kretzschmar D, Kiermayer C, Feldmann P, Hughes DA, Schneuwly S (2003) Deregulation of the Egfr/Ras signaling pathway induces age-related brain degeneration in the Drosophila mutant vap. Mol Biol Cell 14:241-250.

Brown P, Gibbs Jr CJ, Rodgers-Johnson P, Asher DM, Sulima MP, Bacote A, Goldfarb LG, Gajdusek DC (1994) Human spongiform encephalopathy: the National Institutes of Health series of 300 cases of experimentally transmitted disease. Ann Neurol 35:513-529.

Chiesa R, Drisaldi B, Quaglio E, Migheli A, Piccardo P, Ghetti B, Harris DA (2000) Accumulation of protease-resistant prion protein (PrP) and apoptosis of cerebellar granule cells in transgenic mice expressing a PrP insertional mutation. Proc Natl Acad Sci USA 97:5574-5579.

Deleault NR, Lucassen RW, Supattapone S (2003a) RNA molecules stimulate prion protein conversion. Nature 425:717-720.

Deleault NR, Dolph PJ, Feany MB, Cook ME, Nishina K, Harris DA, Supattapone S (2003b) Post-transcriptional suppression of pathogenic prion protein expression in Drosophila neurons. J Neurochem 85:1614-1623.

Driscoll M, Gerstbrein B (2003) Dying for a cause: invertebrate genetics takes on human neurodegeneration. Nat Rev Genet 4:181-194.

Duchen LW, Poulter M, Harding AE (1993) Dementia associated with a 216 base pair insertion in the prion protein gene. Clinical and neuropathological features. Brain 116:555-567.

Feany MB, Bender WW (2000) A Drosophila model of Parkinson's disease. Nature 404:394-398.

Ganetzky B, Flanagan JR (1978) On the relationship between senescence and age-related changes in two wild-type strains of Drosophila melanogaster. Exp Gerontol 13:189-196.

Ghetti B, Tagliavini F, Takao M, Bugiani O, Piccardo P (2003) Hereditary prion protein amyloidoses. Clin Lab Med 23:65-85, viii.

Glatzel M, Stoeck K, Seeger H, Luhrs T, Aguzzi A (2005) Human prion diseases: molecular and clinical aspects. Arch Neurol 62:545-552.

Gray F, Chretien F, Adle-Biassette H, Dorandeu A, Ereau T, Delisle MB, Kopp N, Ironside JW, Vital C (1999) Neuronal apoptosis in Creutzfeldt-Jakob disease. J Neuropathol Exp Neurol 58:321-328.

Hsiao KK, Scott M, Foster D, Groth DF, DeArmond SJ, Prusiner SB (1990) Spontaneous neurodegeneration in transgenic mice with mutant prion protein. Science 250:1587-1590.

Korth C, Stierli B, Streit P, Moser M, Schaller O, Fischer R, Schulz-Schaeffer
W, Kretzschmar H, Raeber A, Braun U, Ehrensperger F, Hornemann S, Glockshuber R, Riek R, Billeter M, Wuthrich K, Oesch B (1997) Prion $(\mathrm{PrPSc})$-specific epitope defined by a monoclonal antibody. Nature 390:74-77.

Krasemann S, Zerr I, Weber T, Poser S, Kretzschmar H, Hunsmann G, Bodemer W (1995) Prion disease associated with a novel nine octapeptide repeat insertion in the PRNP gene. Brain Res Mol Brain Res 34:173-176.

Le Bourg E, Lints FA (1992) Hypergravity and aging in Drosophila melanogaster. 4. Climbing activity. Gerontology 38:59-64.

Manson JC, Jamieson E, Baybutt H, Tuzi NL, Barron R, McConnell I, Somerville R, Ironside J, Will R, Sy MS, Melton DW, Hope J, Bostock C (1999) A single amino acid alteration (101L) introduced into murine PrP dramatically alters incubation time of transmissible spongiform encephalopathy. EMBO J 18:6855-6864.

Marsh JL, Walker H, Theisen H, Zhu YZ, Fielder T, Purcell J, Thompson LM (2000) Expanded polyglutamine peptides alone are intrinsically cytotoxic and cause neurodegeneration in Drosophila. Hum Mol Genet 9:13-25.

Nazor KE, Kuhn F, Seward T, Green M, Zwald D, Purro M, Schmid J, Biffiger K, Power AM, Oesch B, Raeber AJ, Telling GC (2005) Immunodetection of disease-associated mutant $\operatorname{PrP}$, which accelerates disease in GSS transgenic mice. EMBO J 24:2472-2480.

Palmer MS, Collinge J (1993) Mutations and polymorphisms in the prion protein gene. Hum Mutat 2:168-173.

Phelps CB, Brand AH (1998) Ectopic gene expression in Drosophila using GAL4 system. Methods 14:367-379.

Prusiner SB (1982) Novel proteinaceous infectious particles cause scrapie. Science 216:136-144

Prusiner SB (1997) Prion diseases and the BSE crisis. Science 278:245-251.

Raeber AJ, Muramoto T, Kornberg TB, Prusiner SB (1995) Expression and targeting of Syrian hamster prion protein induced by heat shock in transgenic Drosophila melanogaster. Mech Dev 51:317-327.

Rubin GR, Spradling AC (1982) Genetic transformation of Drosophila with transposable element vectors. Science 218:348-353.

Rubin GR, Spradling AC (1983) Vectors for P element-mediated gene transfer in Drosophila. Nucleic Acids Res 11:6341-6351.

Sasaki K, Doh-ura K, Furuta A, Nakashima S, Morisada Y, Tateishi J, Iwaki T (2003) Neuropathological features of a case with schizophrenia and prion protein gene P102L mutation before onset of Gerstmann-Straussler-Scheinker disease. Acta Neuropathol (Berl) 106:92-96.

Tateishi J, Kitamoto T (1995) Inherited prion diseases and transmission to rodents. Brain Pathol 5:53-59.

Tremblay P, Ball HL, Kaneko K, Groth D, Hegde RS, Cohen FE, DeArmond SJ, Prusiner SB, Safar JG (2004) Mutant PrPSc conformers induced by a synthetic peptide and several prion strains. J Virol 78:2088-2099.

Warrick JM, Paulson HL, Gray-Board GL, Bui QT, Fischbeck KH, Pittman RN, Bonini NM (1998) Expanded polyglutamine protein forms nuclear inclusions and causes neural degeneration in Drosophila. Cell 93:939-949.

Wittmann CW, Wszolek MF, Shulman JM, Salvaterra PM, Lewis J, Hutton M, Feany MB (2001) Tauopathy in Drosophila: neurodegeneration without neurofibrillary tangles. Science 293:711-714. 